\title{
Single-Beam 1-Bit Reflective Metasurface Using Pre-Phased Unit Cells for Normally Incident Plane Waves
}

\author{
Jiexi Yin, Student Member, IEEE, Qi Wu, Member, IEEE, Qun Lou, Member, IEEE, \\ Haiming Wang, Member, IEEE, Zhining Chen, Fellow, IEEE, and Wei Hong, Fellow, IEEE
}

\begin{abstract}
A single-beam pre-phased 1-bit reflective metasurface is proposed to achieve single-beam patterns under normally incident plane waves. Theoretical analysis and numerical simulations are presented to show that, under normally incident waves, single-beam patterns can be achieved by introducing a fixed pre-phase distribution with two values in the 1-bit metasurface. Compared with conventional 1-bit reflective metasurfaces, the proposed scheme alleviates the inherent limitation of single-beam patterns on 1-bit reflective metasurfaces under normally incident plane waves. To verify the proposed scheme, a 1-bit unit cell is designed with a $180^{\circ} \pm 25^{\circ}$ phase difference between the two states for frequencies ranging from 34.3 to $49.9 \mathrm{GHz}$, and a layerstacking method is proposed to achieve two pre-phases with a 90-degree phase difference. As an example, three 1-bit reflective metasurfaces comprising $20 \times 20$ unit cells with single beams pointing separately at 0,15 and 30 degrees are designed and measured over frequencies of 37.0 to $41.0 \mathrm{GHz}$; the measured sidelobe levels are less than $\mathbf{- 7 . 8} \mathrm{dB}$. Simulated and measured results show that the proposed pre-phased 1-bit metasurface can achieve single-beam patterns under normally incident plane waves.
\end{abstract}

Index Terms-1-bit, reflective metasurface, single-beam, prephased, normally incident waves.

\section{INTRODUCTION}

$\mathbf{T}$ HE manipulation of electromagnetic (EM) waves has recently attracted substantial attention with the development of metasurfaces as well as the increasing requirements on wireless communications [1]-[4]. One of the challenges facing the millimeter wave (mmWave) is penetration loss,

Manuscript received July 27, 2019; revised Feb. 23, 2020; accepted Date of publication ; date of current . This work was supported in part by the National Key R\&D Program of China under Grant 2018YFB1801101, the National Natural Science Foundation of China under Grants 61671145 and 61960206006, and the Key R\&D Program of Jiangsu Province of China under Grant BE2018121.

J. X. Yin is with the State Key Laboratory of Millimeter Waves and the School of Information Science and Engineering, Southeast University, Nanjing 210096, China, and with the Department of Electrical and Computer Engineering, National University of Singapore, Singapore 117583 (e-mail: yinjiexi@seu.edu.cn).

Q. Lou and Z. N. Chen are with the Department of Electrical and Computer Engineering, National University of Singapore, Singapore 117583 (e-mail: qlou@ieee.org; eleczn@nus.edu.sg).

Q. Wu, H. M. Wang, and W. Hong are with the State Key Laboratory of Millimeter Waves and the School of Information Science and Engineering, Southeast University, Nanjing 210096, China, and with Purple Mountain Laboratories, Nanjing 211111, China (e-mail: qiwu@seu.edu.cn; hmwang@seu.edu.cn; weihong@seu.edu.cn). (Corresponding author: Haiming Wang.)

Color versions of one or more of the figures in this paper are available online at http://ieeexplore.iee.org.

Digital Object Identifier which restricts the development and applications of mmWave indoor communication. Nevertheless, penetration loss can be alleviated if a surface can guide incoming plane waves from one room to another rather than let the waves penetrate through a thick wall. For example, mmWave penetration loss can be avoided or diminished if a series of reflective metasurfaces that can manipulate incoming plane waves toward an arbitrary direction is used to guide those waves without penetrating the concrete walls [5]. Therefore, reflective metasurfaces, which can guide incident plane waves to the desired direction, have excellent potential for indoor mmWave wireless communications.

EM waves can be guided to an arbitrary direction when there are precise phase compensations for each unit cell on the metasurfaces [6]. However, when precise phase compensations are difficult to achieve, discrete phase compensations are used as an alternative to control the radiation beams. One of the most classic discrete phase compensation techniques is based on the use of 1-bit or 2-bit unit cells, which are easily achieved using the ON and OFF states of PIN diodes. Although 2-bit or higher-order-bit unit cells can provide greater designed freedom than 1-bit unit cells for beamforming and beam scanning, particularly in the generation of singlebeam radiation patterns for normally incident plane waves, the cell designs of higher-order-bit unit cells are much more complicated, especially when integrated with beam scanning control systems. Therefore, to simplify the complexity of beam-scanning systems and reduce the fabrication cost, 1-bit metasurfaces with single-beam radiation patterns are preferred.

Accordingly, as a type of reflective metasurface, several 1bit reflectarrays were proposed for beamforming and beam scanning in [7]-[10]. A large electronically reconfigurable reflectarray was designed by a 1-bit unit cell [8]; by using a horn antenna as the feeding source to provide approximately spherical waves, this reflectarray can achieve single-beam patterns. In fact, most single-beam 1-bit reflective metasurfaces developed to date utilize horn antennas to imitate spherical waves as incident waves. The inherent reason for this is that spherical waves can introduce an intrinsic pseudorandom distribution of the phase quantization error, making single beams achievable [11]. However, regarding normally incident plane waves, 1bit metasurfaces can hardly generate a single-beam radiation pattern. In [10], single-beam patterns were realized using a programmable metasurface with a 1-bit unit cell and spherical waves; however, when using normally incident waves, the 


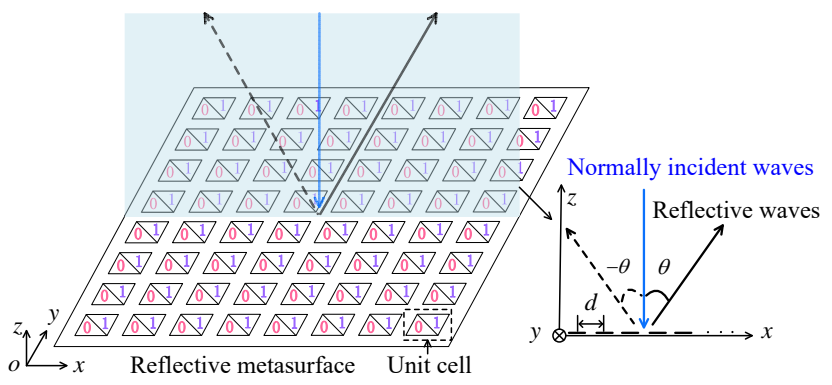

Fig. 1. Geometry of the 1-bit reflective metasurface.

patterns contained two symmetrical beams. Currently, only 2bit or higher-order-bit metasurfaces can produce single-beam patterns for normally incident plane waves [12]-[14].

In this work, a single-beam pre-phased reflective metasurface is presented for normally incident plane waves. First, a theoretical analysis is presented to explain the reason for symmetrical radiation patterns for conventional 1-bit metasurfaces under normally incident plane waves. Next, another analysis is performed to show that the proposed 1-bit metasurface can realize single-beam patterns when the unit cells are properly pre-phased. Finally, as a demonstration, a broadband 1-bit unit cell is proposed, and three pre-phased reflective metasurfaces with different single-beam radiation patterns are designed, fabricated, and measured.

\section{Theoretical Analysis of the 1-Bit Metasurface}

The 1-bit reflective metasurface with $N \times N$ unit cells is shown in Fig. 1. To realize different patterns, the states of 1-bit unit cells are switched between State 0 and State 1; a $\pi$ phase difference can be achieved between these two states. Without loss of generality, State 0 and State 1 of the unit cells are assumed to provide 0 and $\pi$ phase compensations, respectively. That is, the 1-bit phase compensation $\phi_{s, k}$ for the $k$-th column and $s$-th row can be written as

$$
\phi_{s, k}= \begin{cases}0 & \text { State } 0 \\ \pi & \text { State } 1,\end{cases}
$$

where $s, k=1,2, \ldots, N$.

We assume that the 1-bit metasurface is placed in the xoy plane and that the reflective main beam is within the xoz plane, as shown in Fig. 1. Here, $\theta$ is the elevation angle, and the unit cells of the metasurface are uniformly distributed with a spacing $d$. The rows of the array are parallel to the $x$ axis, and the columns are parallel to the $y$ axis.

Let the 1-bit phase compensation be $\phi_{s, k}$ and the initial phase be $\xi_{s, k}$. Considering the progressive phase, the total phase $\varphi_{s, k}$ of the unit cell in the $s$-th row and $k$-th column for the $x o z$ plane is given by

$$
\varphi_{s, k}=\frac{2 \pi k d}{\lambda} \sin \theta+\phi_{s, k}+\xi_{s, k},
$$

where $\lambda$ is the wavelength in free space. For a 1-bit reflective metasurface with uniform amplitude, using trigonometric identities, the modulus of the normalized array factor $\left|\mathrm{AF}_{0}(\theta)\right|$ for the $x o z$ plane can be derived as [15]

$$
\begin{aligned}
& \left|\mathrm{AF}_{0}(\theta)\right| \\
& =\frac{1}{N^{2}}\left|\sum_{s=1}^{N} \sum_{k=1}^{N}\left(\cos \varphi_{s, k}+j \sin \varphi_{s, k}\right)\right| \\
& =\frac{1}{N^{2}}\left(\left(\sum_{s=1}^{N} \sum_{k=1}^{N} \cos \varphi_{s, k}\right)^{2}+\left(\sum_{s=1}^{N} \sum_{k=1}^{N} \sin \varphi_{s, k}\right)^{2}\right)^{\frac{1}{2}} \\
& =\frac{1}{N^{2}}\left(\sum_{a=1}^{N} \sum_{b=1}^{N} \sum_{v=1}^{N} \sum_{w=1}^{N} \cos \varphi_{a, b} \cos \varphi_{v, w}\right. \\
& \left.\quad+\sum_{a=1}^{N} \sum_{b=1}^{N} \sum_{v=1}^{N} \sum_{w=1}^{N} \sin \varphi_{a, b} \sin \varphi_{v, w}\right)^{\frac{1}{2}} \\
& =\frac{1}{N^{2}}\left(\sum_{a=1}^{N} \sum_{b=1}^{N} \sum_{v=1}^{N} \sum_{w=1}^{N} \cos \left(\varphi_{a, b}-\varphi_{v, w}\right)\right)^{\frac{1}{2}} \\
& =\frac{1}{N^{2}}\left(\sum _ { a = 1 } ^ { N } \sum _ { b = 1 } ^ { N } \sum _ { v = 1 } ^ { N } \sum _ { w = 1 } ^ { N } \operatorname { c o s } \left(\frac{2 \pi(b-w) d}{\lambda} \sin \theta\right.\right. \\
& \left.\left.\quad+\left(\phi_{a, b}-\phi_{v, w}\right)+\left(\xi_{a, b}-\xi_{v, w}\right)\right)\right)^{\frac{1}{2}} .
\end{aligned}
$$

where $a$ and $v$ represent the row index and $b$ and $w$ represent the column index.

Under spherical incident waves, the constant-phase surfaces are spherical surfaces, and the initial phases $\xi_{s, k}$ on the reflective metasurface range from 0 to $2 \pi$. Therefore, $-2 \pi \leq \xi_{a, b}-\xi_{v, w} \leq 2 \pi$. In other words, spherical wavesfronts introduce an intrinsic pseudorandom distribution of the phase quantization error [11], and thus, $\left|\mathrm{AF}_{0}(\theta)\right|$ is a non-even function.

Under normally incident plane waves, the constant-phase surfaces are planar, so there are a large number of identical compensated phases for the reflective metasurface, i.e., $\phi_{1, b}=$ $\phi_{2, b}=\cdots=\phi_{N, b} \triangleq \phi_{b}$ and $\phi_{1, w}=\phi_{2, w}=\cdots=\phi_{N, w} \triangleq$ $\phi_{w}$. And the initial phases on the reflective metasurface are the same, i.e., $\xi_{a, b}-\xi_{v, w}=0$ regardless of row and column indices. Consequently, $\left|\mathrm{AF}_{0}(\theta)\right|$ can be simplified as

$$
\begin{aligned}
& \left|\mathrm{AF}_{1}(\theta)\right| \\
& =\frac{1}{N}\left(\sum_{b=1}^{N} \sum_{w=1}^{N} \cos \left(\frac{2 \pi(b-w) d}{\lambda} \sin \theta+\left(\phi_{b}-\phi_{w}\right)\right)\right)^{\frac{1}{2}} .
\end{aligned}
$$

As in (4), the value of $\left(\phi_{b}-\phi_{w}\right)$ can be chosen only among $-\pi, 0$ and $\pi$ for conventional 1-bit metasurfaces under normally incident plane waves. Therefore, $\left|\mathrm{AF}_{1}(\theta)\right|$ is an even function, that is,

$\left|\mathrm{AF}_{1}(\theta)\right|=\frac{1}{N}\left(\sum_{b=1}^{N} \sum_{w=1}^{N}(-1)^{u} \cos \left(\frac{2 \pi(b-w) d}{\lambda} \sin \theta\right)\right)^{\frac{1}{2}}$,

where

$$
u= \begin{cases}0 & \phi_{b}-\phi_{w}=0 \\ 1 & \phi_{b}-\phi_{w}= \pm \pi .\end{cases}
$$


Equations (3) and (5) reveal the inherent difference between spherical incident waves and normally incident plane waves. For spherical incident waves, the intrinsic pseudorandom distribution of the phase quantization error makes (3) a non-even function, and thus, single-beam patterns can be realized. However, for normally incident plane waves, without an intrinsic pseudorandom distribution of the phase quantization error, (3) is simplified to (5). Therefore, the modulus of the array factor is an even function, and there are always two symmetrical beams (not including when the emerging angle is 0 degrees) for conventional 1-bit reflective metasurfaces under normally incident plane waves.

The symmetry of (5) should be broken to achieve singlebeam patterns. Here, we propose the method of introducing the pre-phase $\psi_{s, k}$ for each unit cell composing the metasurface. Assuming that $\Psi=\left(\psi_{s, k}\right)_{N \times N}$ is a pre-phase distribution that is fixed while the 1-bit metasurface switches states, when $\psi_{s, k}$ is added to the total phase $\varphi_{s, k}$, the reflection phase $\varphi_{s, k}^{\prime}$ of the metasurface can be written as

$$
\varphi_{s, k}^{\prime}=\frac{2 \pi k d}{\lambda} \sin \theta+\phi_{s, k}+\psi_{s, k} .
$$

Then,

$$
\begin{array}{r}
\left|\mathrm{AF}_{2}(\theta)\right|=\frac{1}{N^{2}}\left(\sum _ { a = 1 } ^ { N } \sum _ { b = 1 } ^ { N } \sum _ { v = 1 } ^ { N } \sum _ { w = 1 } ^ { N } \operatorname { c o s } \left(\frac{2 \pi(b-w) d}{\lambda} \sin \theta\right.\right. \\
\left.\left.+\left(\phi_{a, b}-\phi_{a, b}\right)+\left(\psi_{v, w}-\psi_{v, w}\right)\right)\right)^{\frac{1}{2}} .
\end{array}
$$

Obviously, the symmetry can be broken when $\psi_{s, k}$ is the same with the initial phase of the spherical waves. However, for spherical waves, this may introduce a complicated structure because its initial phases range from 0 to $2 \pi$.

Two values of pre-phases are sufficient to ensure that equation (7) is a non-even function. Here, two pre-phases with a phase difference of $\pi / 2$ are chosen to obtain the odd terms in (7); then, the symmetry can be broken. Without loss of generality, 0 and $\pi / 2$ are chosen as the two pre-phases. Then, for normally incident plane waves, equation (7) can be simplified as

$$
\left|\mathrm{AF}_{2}(\theta)\right|=\frac{1}{N^{2}}\left(\sum_{a=1}^{N} \sum_{b=1}^{N} \sum_{v=1}^{N} \sum_{w=1}^{N} f(\theta, a, b, v, w)\right)^{\frac{1}{2}},
$$

where

$$
\begin{aligned}
& f(\theta, a, b, u, v)= \\
& \left\{\begin{aligned}
\cos \frac{2 \pi(b-w) d}{\lambda} \sin \theta & \omega_{a, b}-\omega_{v, w}=0, \\
-\cos \frac{2 \pi(b-w) d}{\lambda} \sin \theta & \omega_{a, b}-\omega_{v, w}= \pm \pi \\
\sin \frac{2 \pi(b-w) d}{\lambda} \sin \theta & \omega_{a, b}-\omega_{v, w}=-\frac{\pi}{2}, \frac{3 \pi}{2}, \\
-\sin \frac{2 \pi(b-w) d}{\lambda} \sin \theta & \omega_{a, b}-\omega_{v, w}=\frac{\pi}{2},-\frac{3 \pi}{2},
\end{aligned}\right.
\end{aligned}
$$

with $\omega_{a, b}=\phi_{a, b}+\psi_{a, b}$ and $\omega_{v, w}=\phi_{v, w}+\psi_{v, w}$.

Because $\left|\mathrm{AF}_{2}(\theta)\right|^{2}$ is a sum of several even functions and several odd functions, it is neither an even function nor an odd function. This means that by adding a fixed-phase distribution $\Psi$ to the design of a 1-bit metasurface under normally incident plane waves, the symmetrical patterns of conventional 1-bit metasurfaces can be broken, and single-beam patterns can be realized.

\section{Numerical Design AND Simulation of Pre-PHASED 1-Bit Metasurfaces}

Based on the pre-phase concept described above, we regard each unit cell in the metasurface as a radiation element. Using the theory of antenna synthesis, the pre-phased metasurfaces with $20 \times 20$ unit cells for normally incident plane waves are designed and calculated. The pre-phase distribution $\Psi$ on the metasurface can be obtained by the function

$$
\Psi=\frac{\pi}{2} \operatorname{randi}([0,1], 20,20),
$$

where $\operatorname{randi}([0,1], 20,20)$ represents a $20 \times 20$ matrix with a value of either 0 or 1 for each cell, and all the elements in this matrix are generated by a uniformly distributed pseudorandom function. As a demonstration, the design goals here are set to be a single beam pointing at 0,15 and 30 degrees. The pre-phase distribution $\Psi$ is generated using (10) and is the same in all designs. The spacing between adjacent unit cells is approximately a half-wavelength in free space at the frequency of operation.

For the sake of comparison, the precise phase-compensated and conventional 1-bit metasurfaces are also calculated for each case under normally incident plane waves. The phase distributions for all three phase-compensated methods corresponding to a 30-degree beam direction are illustrated in Fig. 2 , and the patterns of the array factors are shown in Fig. 3(c). The phase distribution on the reflective surface of the precise phase-compensated metasurface displays in Fig. 2(a), which generates a single-beam pattern. The phase distribution on the reflective surface of conventional 1-bit metasurface is symmetrical as shown in Fig. 2(b); hence, $\left|\mathrm{AF}_{0}(\theta)\right|$ becomes an even function, and the pattern is symmetrical with two beams. By introducing pseudorandom uniformly distributed pre-phases, the symmetry of the phase distribution is broken, as shown in Fig. 2(c), and thus, $\left|\mathrm{AF}_{0}(\theta)\right|$ is a non-even function. Considering pre-phases and 1-bit compensation phases, there are four kinds of reflection phases on the proposed reflective metasurface, namely, reflection phases of 0 degrees, 90 degrees, 180 degrees, and 270 degrees, which represent State 0 with a 0 -degree pre-phase, State 1 with a 0 -degree pre-phase, State 0 with a 90-degree pre-phase, and State 1 with a 90-degree pre-phase, respectively. Each pre-phased unit cell can be switched between only two states, and the pre-phase distribution is fixed for different beam directions. Therefore, pre-phased 1-bit metasurfaces are quite different from conventional 2-bit metasurfaces.

Under normally incident plane waves, the calculated patterns in the $x o z$ plane for the three metasurfaces are plotted in Fig. 3, in which the patterns for the conventional 1-bit and precise phase-compensated metasurfaces are presented for comparison. The sidelobe levels (SLLs) and beam directions of the patterns in the xoz plane are presented in Table I. It is obvious that the undesirable symmetrical beam of the conventional 1-bit metasurface is effectively suppressed by 


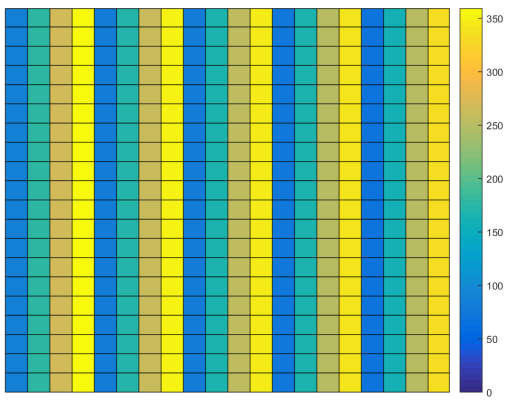

(a)

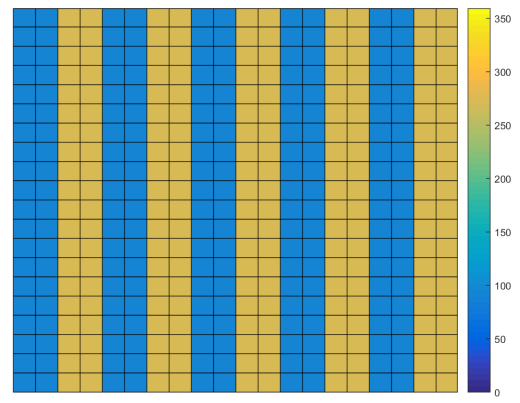

(b)

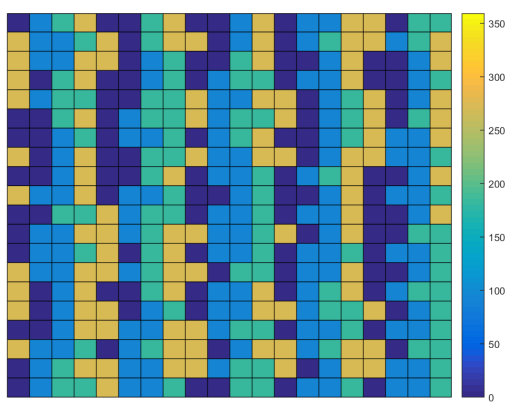

(c)

Fig. 2. Calculated phase distributions for the three phase-compensated methods under normally incident waves, for 30-degree beam direction: (a) precise phase compensation, (b) conventional 1-bit phase compensation, and (c) pre-phased 1-bit phase compensation.

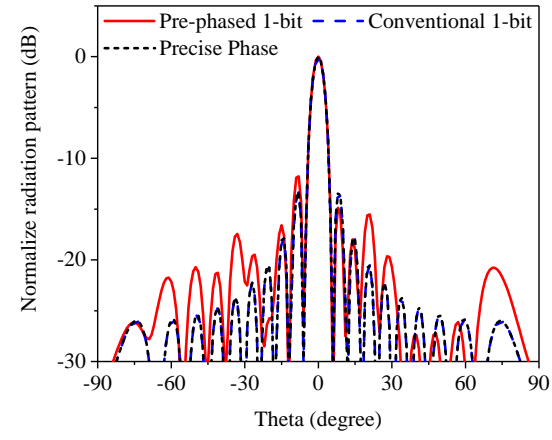

(a)

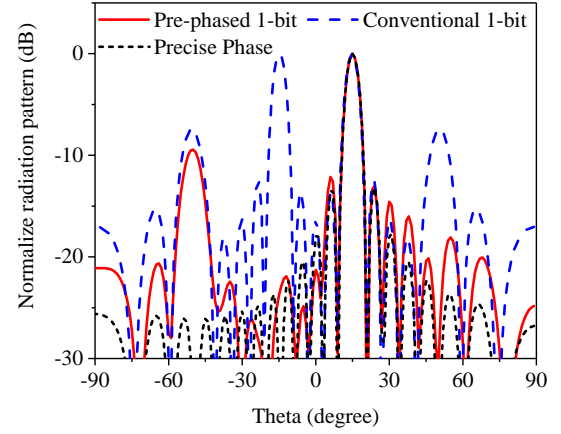

(b)

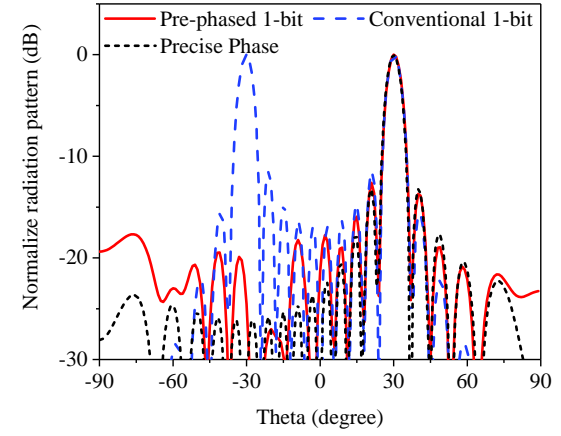

(c)

Fig. 3. Calculated patterns under normally incident plane waves in the $x o z$ plane: (a) 0 degrees, (b) 15 degrees, and (c) 30 degrees.

TABLE I

Simulated Performance of the Proposed Pre-PHASEd 1-Bit, Conventional 1-BIT, and PRECISE Phase-COMPENSATED Metasurfaces Under Normally InCident Plane WaVes.

\begin{tabular}{cccc}
\hline \hline & Pre-phase & Conventional & Precise phase \\
\hline Direction $\left({ }^{\circ}\right)$ & $0 / 15 / 30$ & $0 / \pm 15 / \pm 30$ & $0 / 15 / 30$ \\
\hline SLL $(\mathrm{dB})$ & $-11.8 /-9.4 /-12.6$ & $-13.3 /-7.5 /-11.4$ & $-13.3 /-13.2 /-12.6$ \\
\hline
\end{tabular}

introducing a fixed pre-phase distribution with only two values. Furthermore, a 1-bit metasurface with a single-beam pattern for any other directions can also be designed with the help of a fixed $\Psi$. For a fixed pre-phase distribution, high SLLs may appear in some beam directions during the beam scanning design. However, to optimize and suppress the SLL, different reference phases can be introduced during each designed beam direction [9]. The proposed pre-phased method can also break the symmetry of the array factor under obliquely incident plane waves; consequently, single-beam patterns can also be realized under obliquely incident waves.

\section{Design AND PRototype}

\section{A. 1-Bit Unit Cell}

The configuration of the proposed 1-bit unit cell is illustrated in Fig. 4. For State 0, the unit cell can be regarded as a square patch with a cross-shaped slot in its center. This cross-shaped slot cuts the square patch into four small square patches that are symmetrical with respect to the center of the unit cell for State 0, as shown in Fig. 4(a). In contrast, for State 1 , the four small square patches are connected by four stubs,

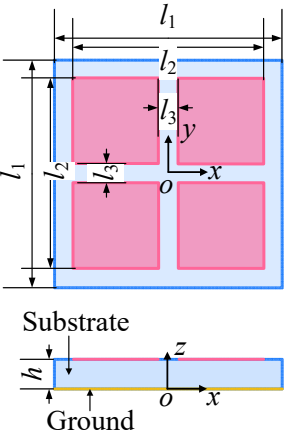

(a)

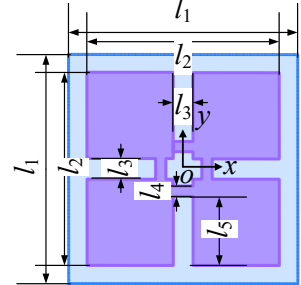

Substrate

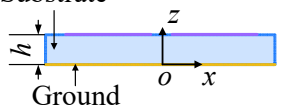

(b)
Fig. 4. Top and side views of the proposed 1-bit unit cell: (a) State 0 and (b) State 1 , where $l_{1}=3.80, l_{2}=3.15, l_{3}=0.30, l_{4}=0.15, l_{5}=1.03$, and $h=0.762$ (Unit: $\mathrm{mm}$ ).

as shown in Fig. 4(b). The difference between the two states is whether the four small patches are connected. Because the unit cell has 90-degree rotational symmetry with respect to its center, the proposed unit cell can respond to both vertically polarized waves and horizontally polarized waves. A Taconic TLY laminate with a dielectric constant of $\varepsilon_{r}=2.2$ is used as the substrate. The designed frequency is in the Q-band and the period of the unit cell is chosen as approximately half the wavelength at $39.0 \mathrm{GHz}$. The simulated performance of the proposed 1-bit unit cell is plotted in Fig. 5. Furthermore, $l_{2}$ and $l_{3}$ are the same in both states; thus, this unit cell can be reconfigured by substituting the four small stubs with four PIN diodes. 


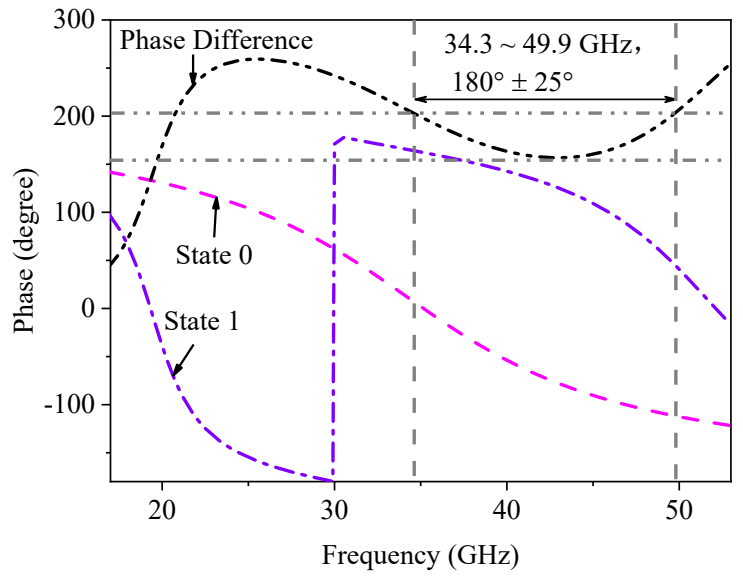

Fig. 5. Simulated performance of the proposed 1-bit unit cell.
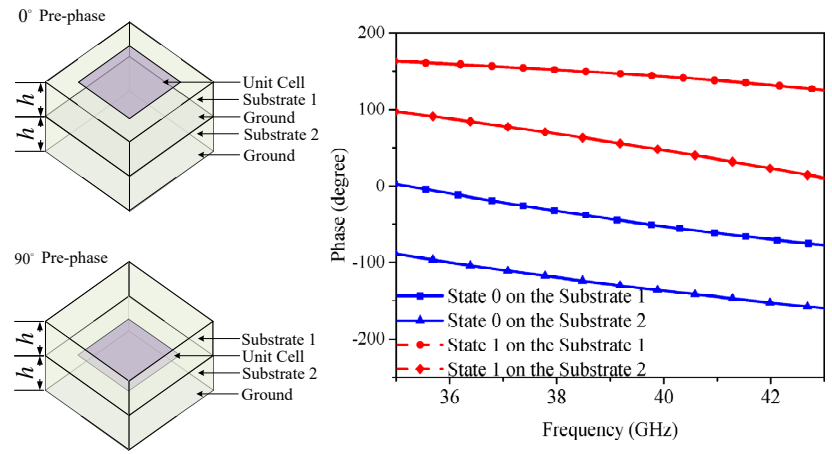

Fig. 6. Reflection phases for the two states on the different substrates with $h=0.762 \mathrm{~mm}$.

\section{B. Pre-Phase Design}

To achieve two pre-phases with a pre-phase difference of approximately 90 degrees, as discussed in Section II, the unit cells are placed on different layers of two stacked substrates, as shown in Fig. 6. The path difference for the incident and reflected waves will produce a pre-phase difference of approximately 90 degrees if the thickness of substrate 1 is oneeighth of a wavelength in the substrate. Considering the limit on the thickness of a printed circuit board (PCB), the thickness of the substrate is chosen as $0.762 \mathrm{~mm}$. The reflection phases of the unit cells with the pre-phases are shown in Fig. 6, which demonstrates that a 90-degree reflection phase difference can be achieved for the same state and different pre-phases, whereas a 180-degree reflection phase can be achieved for different states and the same pre-phase.

However, the layer-stacking method mentioned above will increase the complexity of the reconfigurable design, so the bias circuit should be carefully designed for reconfigurable pre-phased metasurfaces.

\section{C. $20 \times 20$ Pre-Phased Metasurface}

Based on the proposed 1-bit unit cell and pre-phase design, three $20 \times 20$ pre-phased 1-bit reflective metasurfaces with single beams pointing at 0,15 , and 30 degrees are designed with a center frequency of $39.0 \mathrm{GHz}$. The pre-phase distribution shown in Fig. 2(c), which is generated by a pseudorandom

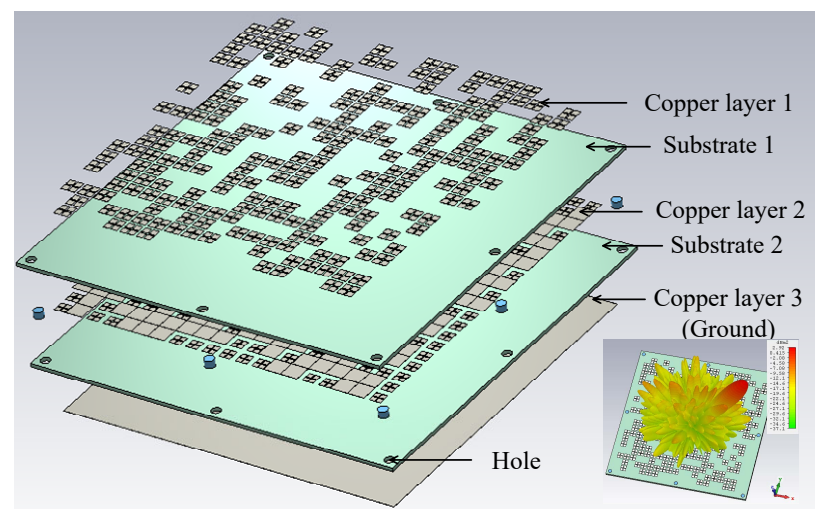

Fig. 7. Geometry of the proposed pre-phased 1-bit metasurface and the array pattern under normally incident plane waves, 30-degree beam direction.

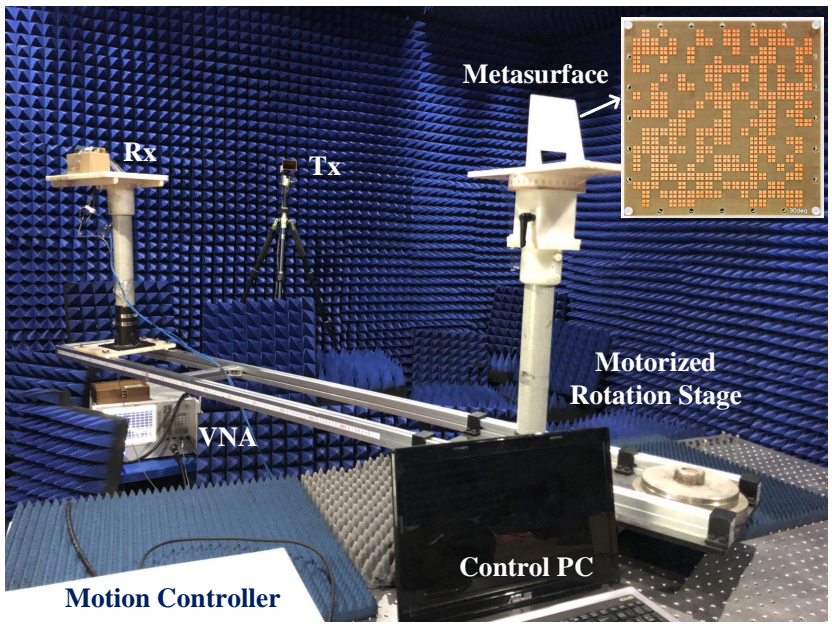

Fig. 8. Measurement setup for the metasurface prototypes.

uniformly distributed function, is utilized for all designed beam directions in this study. After the pre-phase is introduced to the unit cell, the state of each unit cell can be determined by the required phase compensation for the specific beam direction. If the required phase compensation lies in the range from -90 to 90 degrees, State 0 is selected; if the required phase compensation ranges from 90 to 270 degrees, State 1 is selected.

For the 30-degree reflective metasurface, the phase compensations of the unit cells with the pre-phases are shown in Fig. 2(c). The geometry of the metasurface and the pattern are shown in Fig. 7; the metasurface includes two substrates and three copper layers. The Taconic TLY laminate is used for the metasurface design. A single beam with a suppressed SLL is achieved in the 3D space. The simulated and measured patterns are shown in Fig. 9, and the performance of the designed metasurfaces is presented in Table II. The patterns are degraded at the sideband frequencies because the target frequency is $39.0 \mathrm{GHz}$. Fortunately, some methods can be used to alleviate this degradation for further optimization [16], [17].

\section{RESUlts AND DISCUSSION}

\section{A. Measured Results}

To verify the performance of the proposed 1-bit pre-phased metasurface under normally incident plane waves, three re- 
TABLE II

Simulated Performance of Three Pre-Phased 1-Bit Metasurfaces $\left(0^{\circ} / 15^{\circ} / 30^{\circ}\right)$ Under Normally Incident Plane Waves (Vertical POLARIZATION).

\begin{tabular}{ccccc}
\hline \hline Frequency $(\mathrm{GHz})$ & 37.0 & 39.0 & 41.0 & PEC@39.0 \\
\hline Beam Direction $\left(^{\circ}\right)$ & $0 / 15.2 / 32.0$ & $0 / 14.6 / 30.0$ & $0 / 14.0 / 28.5$ & 0 \\
\hline SLL $(\mathrm{dB})(x o z$ plane $)$ & $-13.2 /-12.2 /-9.8$ & $-12.3 /-12.0 /-12.1$ & $-11.8 /-10.0 /-11.2$ & -13.4 \\
\hline 3-dB Beamwidth $\left(^{\circ}\right)$ & $5.1 / 5.8 / 6.0$ & $4.6 / 5.2 / 5.3$ & $4.6 / 4.9 / 5.9$ & 5.0 \\
\hline Normalized Max. RCS $(\mathrm{dB})$ & $-1.3 /-4.1 /-5.9$ & $-2.1 /-4.2 /-5.5$ & $-2.2 /-4.5 /-5.4$ & 0 \\
\hline \hline
\end{tabular}

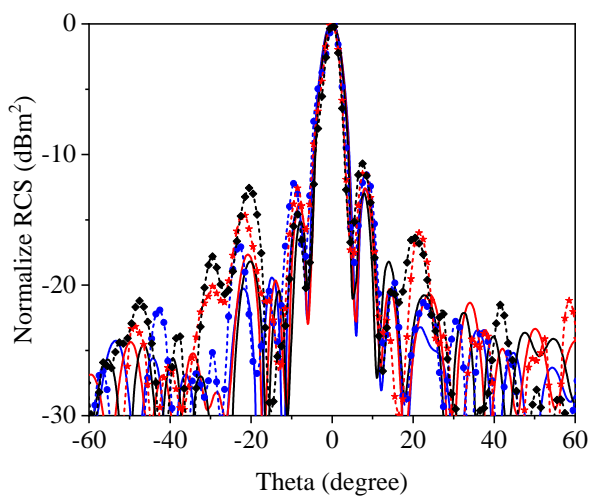

(a)

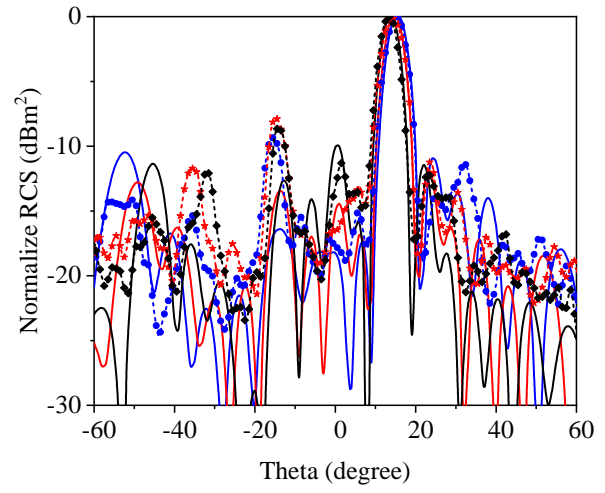

(b)

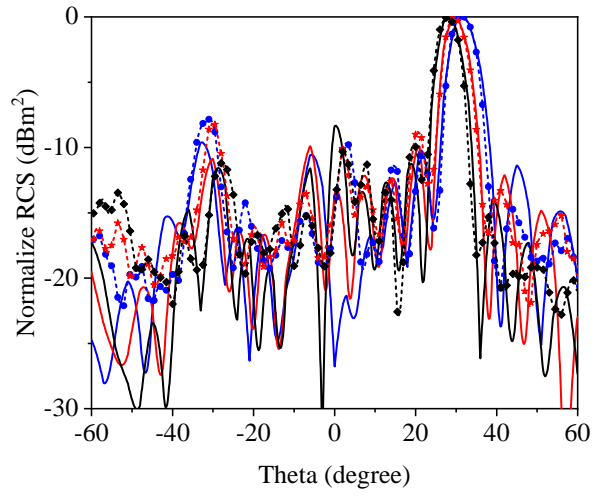

(c)

Sim.@ $37.0 \mathrm{GHz}$ — Sim.@39.0 GHz Sim.@ $41.0 \mathrm{GHz}$

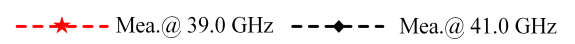

Fig. 9. Simulated and measured patterns of the proposed 1-bit pre-phased metasurface in the xoz plane under normally incident plane waves (vertical polarization): (a) 0 degrees, (b) 15 degrees, and (c) 30 degrees.

flective metasurfaces with single beams pointing separately at 0,15 , and 30 degrees are fabricated. The 1-bit metasurface includes three copper layers and two substrate layers. The two substrate layers with their respective copper layers are separately fabricated using PCB technology and assembled using plastic screws. The measurement setup is presented in Fig. 8. The Tx horn antenna is fixed, and the aperture of the Tx horn is parallel to the aperture of the sample to provide approximate incident plane waves. The $\mathrm{Rx}$ horn antenna is aimed at the center of the sample and is fixed on the motorized rotating stage, which can rotate around the sample; furthermore, a vector network analyzer (VNA) is employed to record the electric field level of the Rx horn from -60 to 60 degrees in the horizontal plane. To reduce the shielding effect during the 0-degree test, the Tx horn is slightly higher than the Rx horn. A control PC is utilized to operate the motion controller and obtain the data from the VNA.

A copper plate of the same size was also measured as a reference; accordingly, the radar cross sections (RCS) of the three metasurfaces are given in Table III in comparison with the RCS of the copper plate. Moreover, the simulated and measured patterns in the xoz plane under normally incident waves are shown in Fig. 9. Single beams can also be obtained under horizontal polarization because the structure of the proposed unit cell is symmetrical. The measured beam directions of the 1-bit pre-phased metasurfaces agree with the designed directions, and the measured SLLs in the xoz plane are suppressed below $-7.8 \mathrm{~dB}$. In addition, the simulated and measured results of the $3-\mathrm{dB}$ beamwidth also agree well.
TABLE III

RCSS AT 39.0 GHz UNDER NoRMALly InCIDENT Plane WaVES.

\begin{tabular}{ccccc}
\hline \hline Sample & PEC & 0-degree & 15-degree & 30-degree \\
\hline Sim. $(\mathrm{dB})$ & 0 & -2.1 & -4.2 & -5.5 \\
\hline Mea. $(\mathrm{dB})$ & 0 & -2.1 & -4.5 & -5.6 \\
\hline Sim.-Mea. $\mid(\mathrm{dB})$ & 0 & 0 & 0.3 & 0.1 \\
\hline \hline
\end{tabular}

\section{B. Comparison and Discussion}

The reported performance of the 1-bit and 2-bit reflective metasurfaces is summarized in Table IV. The supported polarizations in the table indicate the polarization of the incident waves. [8], [9] designed a 1-bit reflective metasurface with single-beam patterns by using a horn antenna to provide spherical waves. [4], [10], [18] designed a 1-bit reflective metasurface with symmetrical patterns under approximate plane waves. Because symmetrical patterns introduce two symmetrical beams, [12], [14] employed 2-bit or higher-order-bit metasurfaces to achieve single-beam patterns under normally incident plane waves. Compared with the 2-bit metasurface, the 1-bit pre-phased metasurface has a lower gain because of the larger phase quantization error but features a lower complexity, especially regarding the reconfigurable beam design. The pre-phased method proposed in this work alleviates the inherent limitation of existing 1-bit reflective metasurfaces, which achieves single-beam patterns under normally incident plane waves. Moreover, the proposed method is simple, and the proposed metasurface has low complexity and a low-cost fabrication process. 
TABLE IV

Comparisons Between the Proposed 1-Bit Metasurface and the Metasurfaces in the References.

\begin{tabular}{cccccccc}
\hline \hline Ref. & Unit Cell & Incident Wave & Unit Cell Num. & Freq. (GHz) & Single Beam & Supported Polarization & Reconfigurability \\
\hline$[4]$ & 1 bit & Plane & Not Given & 10.0 & No & V and H & Not realized \\
\hline$[8]$ & 1 bit & Spherical & $160 \times 160$ & 60.0 & Yes & V and H & Realized \\
\hline$[9]$ & 1 bit & Spherical & $10 \times 10$ & 12.5 & Yes & V or H & Realized \\
\hline$[10]$ & 1 bit & Plane & $40 \times 40$ & 11.1 & No & V or H & Realized \\
\hline$[18]$ & 1 bit & Plane & $8 \times 8$ & 90.0 & No & V and H & Not realized \\
\hline$[12]$ & 2 bit & Plane & Not Given & 10.5 & Yes & V and H & Realized \\
\hline$[14]$ & 2 bit & Plane & $16 \times 16$ & 7.2 & Yes & V or H & Realized \\
\hline This work & 1 bit & Plane & $20 \times 20$ & 39.0 & Yes & V and H & Not realized \\
\hline \hline
\end{tabular}

\section{CONCLUSION}

A pre-phased 1-bit reflective metasurface has been proposed to achieve single-beam patterns under normally incident plane waves. The proposed scheme alleviates the inherent limitation of existing 1-bit reflective metasurfaces under normally incident plane waves, and a pseudorandom uniformly distributed pre-phase is added to the metasurface to break the symmetry of the array factor. A numerical simulation has illustrated that under plane incident waves, single-beam patterns can be achieved by a 1-bit pre-phased metasurface. To design and realize the pre-phased 1-bit metasurface, a broadband 1-bit unit cell has been proposed, and a pre-phased method has been presented to realize two pre-phases with a 90-degree phase difference. To verify the proposed approach, three pre-phased 1-bit metasurfaces under normally incident plane waves with different beam directions have been designed and fabricated via the PCB process. The simulated and measured results have revealed that the proposed 1-bit pre-phased reflective metasurface can achieve single-beam patterns under normally incident plane waves. For further beam scanning and reconfigurable design purposes, the basic circuit should be carefully designed because of the multilayer pre-phased metasurface. Ultimately, the proposed pre-phased method has the potential to achieve beamforming and beam scanning for mmWave indoor communications.

\section{REFERENCES}

[1] Results of the first session of the Conference Preparatory Meeting for WRC-19 (CPM19-1). document CA/226, ITU-R Administrative Circular, Dec. 2015.

[2] T. S. Rappaport, S. Sun et al., "Millimeter wave mobile communications for 5G cellular: It will work!" IEEE Access, vol. 1, pp. 335-349, 2013.

[3] S. V. Hum and J. Perruisseau-Carrier, "Reconfigurable reflectarrays and array lenses for dynamic antenna beam control: A review," IEEE Trans. Antennas Propag., vol. 62, no. 1, pp. 183-198, 2014.

[4] S. Liu and T. J. Cui, "Concepts, working principles, and applications of coding and programmable metamaterials," Adv. Opt. Mater, vol. 5, no. 22, p. 1700624, 2017.
[5] D. R. Smith, V. R. Gowda, O. Yurduseven, S. Larouche, G. Lipworth, Y. Urzhumov, and M. S. Reynolds, "An analysis of beamed wireless power transfer in the fresnel zone using a dynamic, metasurface aperture," J. Appl. Phys., vol. 121, no. 1, p. 014901, 2017.

[6] J. Huang, "Reflectarray antenna," Hoboken-Piscataway, NJ, USA: WileyIEEE Press, 2007.

[7] S. Tian, H. Liu, and L. Li, "Design of 1-bit digital reconfigurable reflective metasurface for beam-scanning," Appl. Sci., vol. 7, no. 9, p. 882, 2017.

[8] H. Kamoda, T. Iwasaki, J. Tsumochi, T. Kuki, and O. Hashimoto, "60-GHz electronically reconfigurable large reflectarray using singlebit phase shifters," IEEE Trans. Antennas Propag., vol. 59, no. 7, pp. 2524-2531, 2011.

[9] H. Yang, F. Yang, S. Xu, Y. Mao, M. Li, X. Cao, and J. Gao, "A 1-bit $10 \times 10$ reconfigurable reflectarray antenna: Design, optimization, and experiment," IEEE Trans. Antennas Propag., vol. 64, no. 6, pp. 22462254, 2016.

[10] H. Yang, X. Cao, F. Yang, J. Gao, S. Xu, M. Li, X. Chen, Y. Zhao, Y. Zheng, and S. Li, "A programmable metasurface with dynamic polarization, scattering and focusing control," Sci. Rep., vol. 6, p. 35692, 2016.

[11] H. Yang, F. Yang, S. Xu, M. Li, X. Cao, J. Gao, and Y. Zheng, "A study of phase quantization effects for reconfigurable reflectarray antennas," IEEE Antennas and Wireless Propag. Lett., vol. 16, pp. 302-305, 2016.

[12] T. J. Cui, M. Q. Qi, X. Wan, J. Zhao, and Q. Cheng, "Coding metamaterials, digital metamaterials and programmable metamaterials," Light: Sci. Appl., vol. 3, no. 10, p. e218, 2014.

[13] T.-J. Cui, S. Liu, and L.-L. Li, "Information entropy of coding metasurface," Light: Sci. Appl., vol. 5, no. 11, p. e16172, 2016.

[14] C. Huang, B. Sun, W. Pan, J. Cui, X. Wu, and X. Luo, "Dynamical beam manipulation based on 2-bit digitally-controlled coding metasurface," Sci. Rep., vol. 7, p. 42302, 2017.

[15] C. A. Balanis, Antenna theory: analysis and design. John wiley \& sons, 2016.

[16] Y. Mao, S. Xu, F. Yang, and A. Z. Elsherbeni, "A novel phase synthesis approach for wideband reflectarray design," IEEE Trans. Antennas Propag., vol. 63, no. 9, pp. 4189-4193, 2015.

[17] Y. Liu, Y. J. Cheng, X. Y. Lei, and P. F. Kou, "Millimeter-wave singlelayer wideband high-gain reflectarray antenna with ability of spatial dispersion compensation," IEEE Trans. Antennas Propag., vol. 66, no. 12 , pp. $6862-6868,2018$.

[18] M. K. T. Al-Nuaimi, Y. He, and W. Hong, "Design of 1-bit coding engineered reflectors for EM-wave shaping and RCS modifications," IEEE Access, vol. 6, pp. 75 422-75 428, 2018. 